\section{How to get out}

Nuclear transport is coordinated by nuclear Ran GTP, which releases cargo from importin proteins and promotes cargo binding to exportin proteins. The role of Ran 'GTP in cargo release is reasonably well understood. But, how does Ran 'GTP function in nuclear protein export to promote cargo binding? M atsuura and Stewart now provide insights by describing the 2.0- $\AA$ resolution crystal structure of a nuclear export complex in Nature.

Thenuclear export complex comprised Ran GTP, Csel (a yeast exportin) and Kap60. Kap60 is an adaptor protein (yeast importin- $\alpha$ ) that binds to nuclear localization signal (NLS)-containing cargo proteins and links them to Kap95 (yeast importin- $\beta$ ) in the cytoplasm. Following protein import, Kap60 is exported from thenucleus as $C$ sel cargo. A notable feature of thestructure - which is

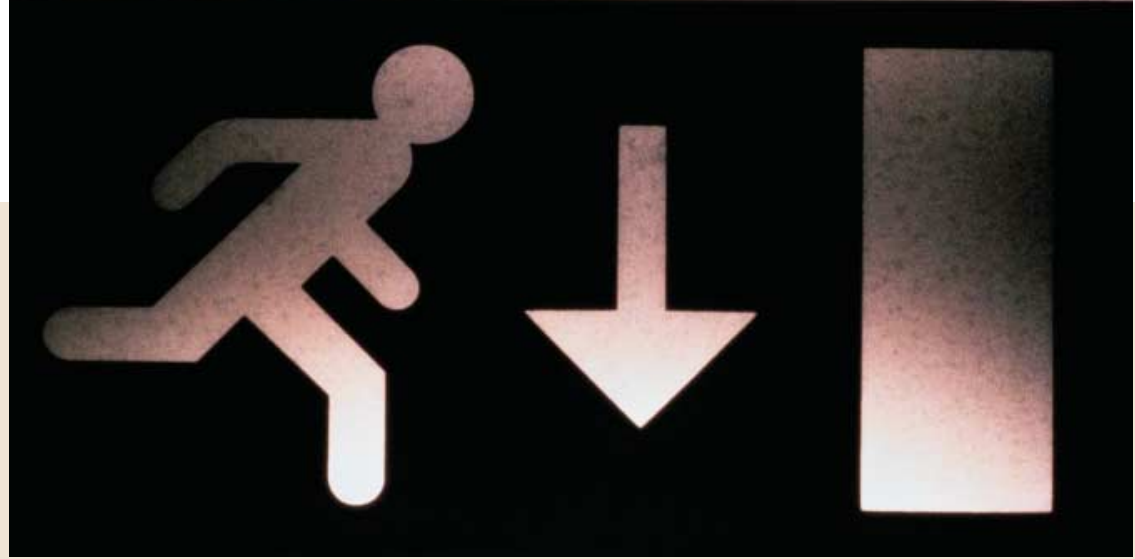

different to that seen for nuclear import complexes- is that C sel surrounds Ran GTP and Kap60. C sel interacts with Ran -GTP at two distinct sites, effectively locking it in the GTP-bound state. The Kap95-binding domain of Kap60 is also ableto bind to theN LSbinding sites of Kap60 in an autoinhibitory manner and, in thenuclear export complex, this domain is clamped to the Kap60 NLSbinding sites by Ran 'GTP and C sel. The fact that this intramolecular interaction in Kap60 is required for $\mathrm{C}$ sel binding ensures that only cargo-free Kap60 is exported.

Mutagenesis studies confirmed the importance of Kap95-binding-domain interactions in nuclear-export-complex assembly and Kap60 export. In addition, the study of other mutant proteins indicated that free C sel adopts a different conformation to that seen in the nuclear export complex. It seems that Kap 60 binding distorts $C$ sel into a high-energy, strained conformation, such that the nuclear export complex is springloaded for disassembly following GTP hydrolysis in the cytoplasm.

Rachel Smallridge

(i) References and links

ORIGINAL RESEARCH PAPER Matsuura, $Y$ \& S Stewart, M. Structural basis for the assembly of a nuclear export complex. Nature 432, 872-877 (2004)

FURTHER READING Hoelz, A. \& Blobel, G. Cell biology: popping out of the nucleus. Nature 432, 815-816 (2004) WEB SITE

Murray Stewart's laboratory:

http://www2.mrc-Imb.cam.ac.uk/groups/ms/

\section{Erasing the mark}
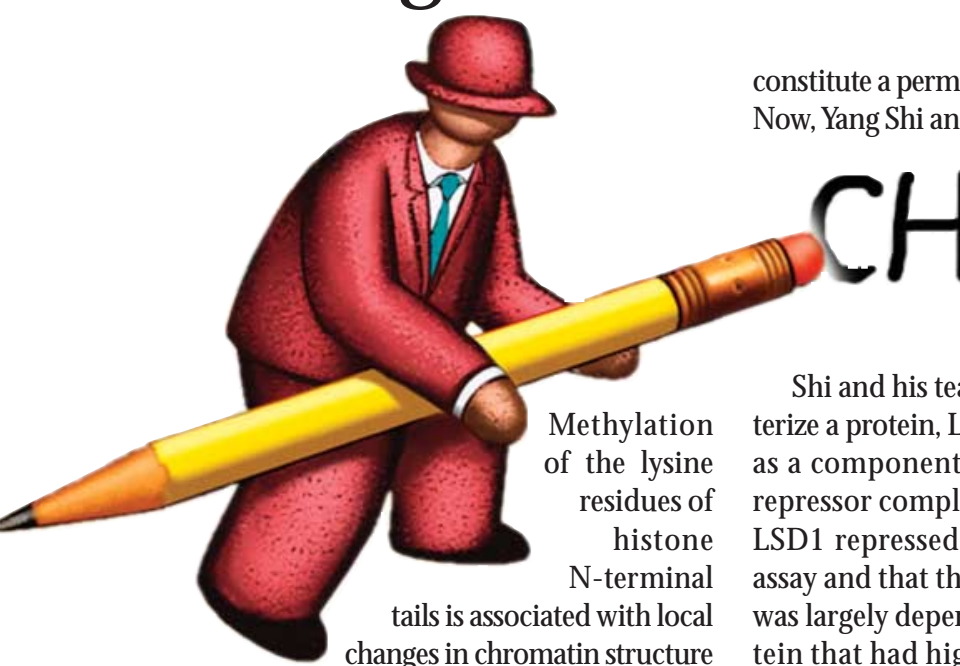
3

M ethylation of the lysine residues of

histone

$\mathrm{N}$-terminal

tails is associated with local changes in chromatin structure and correlates with both gene activation and generepression. The regulation of genes is, however, a dynamic process, and cells must therefore possess the ability to rapidly generate and erase the 'methyl mark'. Whereas several histone methyltransfer ases have been documented, the identification of an enzyme that removes the methyl group from histone tails has remained elusive, which has fuelled the debate that histone methylation might constitutea permanent epigenetic modification. Now, Yang Shi and co-workers have brought us onestep closer to resolving this question by theidentification of a histone lysine demethylase, and they report thisfinding in Cell.

Shi and his team initially set out to characterize a protein, LSD1, that had been identified as a component of several transcriptionalrepressor complexes. First, they showed that LSD 1 repressed gene activity in a reporter assay and that thistranscriptional repression was largely dependent on a region of the protein that had high sequence homology with amine oxidases - metabolic enzymes that are putative histone demethylases. This indicated that LSD 1 might repress transcription by catalysing the demethylation of core histones.

Direct demethylation assays confirmed that LSD 1 removed the methyl group from dimethyl and monomethyl histone $\mathrm{H} 3-\mathrm{K} 4$, but not from trimethyl $\mathrm{H} 3-\mathrm{K} 4, \mathrm{H} 3-\mathrm{K} 9$ or several other methylated lysine and arginine residues. $\mathrm{H} 3-\mathrm{K} 4$ methylation correlates with transcriptional activity, and so it is appropriate that a transcriptional repressor would erase this modification.

Because of its homology to the amine oxidases, the researchers predicted that an LSD 1-mediated demethylation reaction would gener ate formal dehyde. Indeed, when LSD 1 was incubated with dimethylated substratein a spectrophotometric assay, large amounts of formal dehyde were detected. This finding provided further compelling evidence that LSD1 was a bona fide demethylase.

But does LSD 1 repress endogenous genes by catalysing histone demethylation?Theenzymeis found in the Co-REST complex - a transcriptional repressor that silences neuron-specific genes. LSD 1-deficient HeLa cells that were generated by RNA interference aberrantly expressed several neuron-specific target genes, and chromatin-immunoprecipitation analyses detected both a loss of association of LSD 1 and an increase in $\mathrm{H} 3-\mathrm{K} 4$ methylation at the promoters of these derepressed genes.

So, it seems that a lysine demethylase has at last been pinned down. It is important to note, however, that an enzyme that removes the methyl marks that identify heritably silenced chromatin still defies detection. .

Shannon Amoils

(2) References and links

ORIGINAL RESEARCH PAPER Shi, Y. et al. Histone

demethylation mediated by the nuclear amine oxidase homolog LSD1. Cell 119, 941-953 (2004) 